\title{
Long term outcome of brain manganese deposition in patients on home parenteral nutrition
}

Yota Kafritsa, John Fell, Sarah Long, Michael Bynevelt, Wendy Taylor, Peter Milla

\begin{abstract}
Manganese intoxication has been described in children on long term parenteral nutrition presenting with liver and nervous system disorders. Cases are reported of a brother and sister on long term parenteral nutrition with hypermanganesaemia and basal ganglia manganese deposition, detected by magnetic resonance imaging (MRI), without overt neurological signs. Following reduction of manganese intake, basal ganglia manganese was monitored by repeated MRI, and neurological and developmental examinations. An MRI intensity index of the globus pallidus declined over a three year period from 0.318 and 0.385 to 0.205 and 0.134 with concomitant falls in whole blood manganese from 323 and 516 to 226 and $209 \mathrm{nmol} / 1$ (normal range, 73-210 $\mathrm{nmol} / 1$ ). Unlike adult experience these children developed normally without neurological signs. In conclusion, deposited manganese is removed from neural tissue over time and the prognosis is good when neurological manifestations and liver disease are absent.
\end{abstract}

(Arch Dis Child 1998;79:263-265)

Keywords: manganese; parenteral nutrition; basal ganglia; magnetic resonance imaging

\section{Department of Gastroenterology, Great Ormond Street Hospital for Children NHS Trust, London WC1N 3JH, UK \\ Y Kafritsa \\ J Fell \\ S Long \\ P Milla}

Department of Neuroradiology, Great Ormond Street Hospital for Children NHS Trust M Bynevelt

W Taylor

Correspondence to: Dr P Milla, Paediatric Gastroenterology Unit, Institute of Child Health, 30 Guilford Street, London WC1N 1EH, UK.

Accepted 13 March 1998
Manganese toxicity has been described in children receiving long term parenteral nutrition manifest by movement disorders and cholestatic liver disease when parenteral manganese supplementation has been excessive. ${ }^{1-3}$ Increased signal intensity on magnetic resonance imaging (MRI) within the basal ganglia has been observed, particularly in children with movement disorders, which has the characteristics of manganese deposition. ${ }^{4}$ Although the resultant abnormalities of the basal ganglia may be partly reversible in adults, the long term effect on the developing brain of children is unknown. $^{25}$ We have previously described manganese deposition of the basal ganglia in four of 57 children receiving parenteral nutrition for longer than two years. ${ }^{4}$ In this report, we describe the natural history of manganese deposition in the basal ganglia detected by MRI in two of these children following the introduction of reduced manganese supplementation.

\section{Case 1}

A 9 year old boy had been born to a 25 year old primigravida after a normal pregnancy. He was initially breast fed but during the first month of life he developed intractable diarrhoea when changed to formula milk. At 4 months of age he was admitted to the Hospital for Sick Children in London, UK where he was found to have an enteropathy of unknown cause. Dietary exclusion and immunosuppressive treatment were unsuccessful. Total parenteral nutrition was introduced as he could not tolerate any oral feeding. After a prolonged hospital admission he was discharged dependent on parenteral nutrition, tolerating only $30 \mathrm{kcal} / \mathrm{kg} /$ day of nutrients enterally. He progressed satisfactorily on home parenteral nutrition, growing and gaining weight between the 10 th and 25 th centile. Liver function and serum concentrations of trace elements other than manganese remained in the normal range. In 1994 as part of a survey to understand the prevalence of hypermanganesaemia in children receiving long term parenteral nutrition, whole blood manganese concentrations were measured using atomic absorption spectrophotometry. ${ }^{2}$ He had a raised blood manganese concentration of $323 \mathrm{nmol} / 1$ (reference range, 73$210 \mathrm{nmol} / \mathrm{l})$. A standard regimen of home parenteral nutrition had been administered, which contained a trace element solution (Pedel, Kabi Pharmacia Ltd, Milton Keynes, UK) calculated to provide a normal intake of zinc but it then provided $0.2 \mathrm{mmol} / \mathrm{kg}$ of manganese per day. The total duration of parenteral nutrition was 63 months. Cranial MRI was performed to detect any manganese deposition in the brain tissue. T1 weighted images demonstrated bilateral and symmetrical high signal lesions at the basal ganglia, especially in the globus pallidus and the subthalamic nuclei; T2 weighted images did not show any abnormality. These findings were suggestive of the deposition of a paramagnetic element and were consistent with manganese overload. Despite the radiological abnormalities, the child did not have any neurological signs or symptoms and his developmental assessment was normal. A different trace element solution (Peditrace, Kabi Pharmacia Ltd) was introduced into the parenteral nutrition, which contains a lower amount of manganese $(0.01 \mathrm{mmol} / \mathrm{kg} /$ day $)$ according to the American Society for Clinical Nutrition recommendations. ${ }^{6}$ The concentrations of all the other components of the parenteral nutrition solution were unaltered. Following the reduction in manganese admin- 

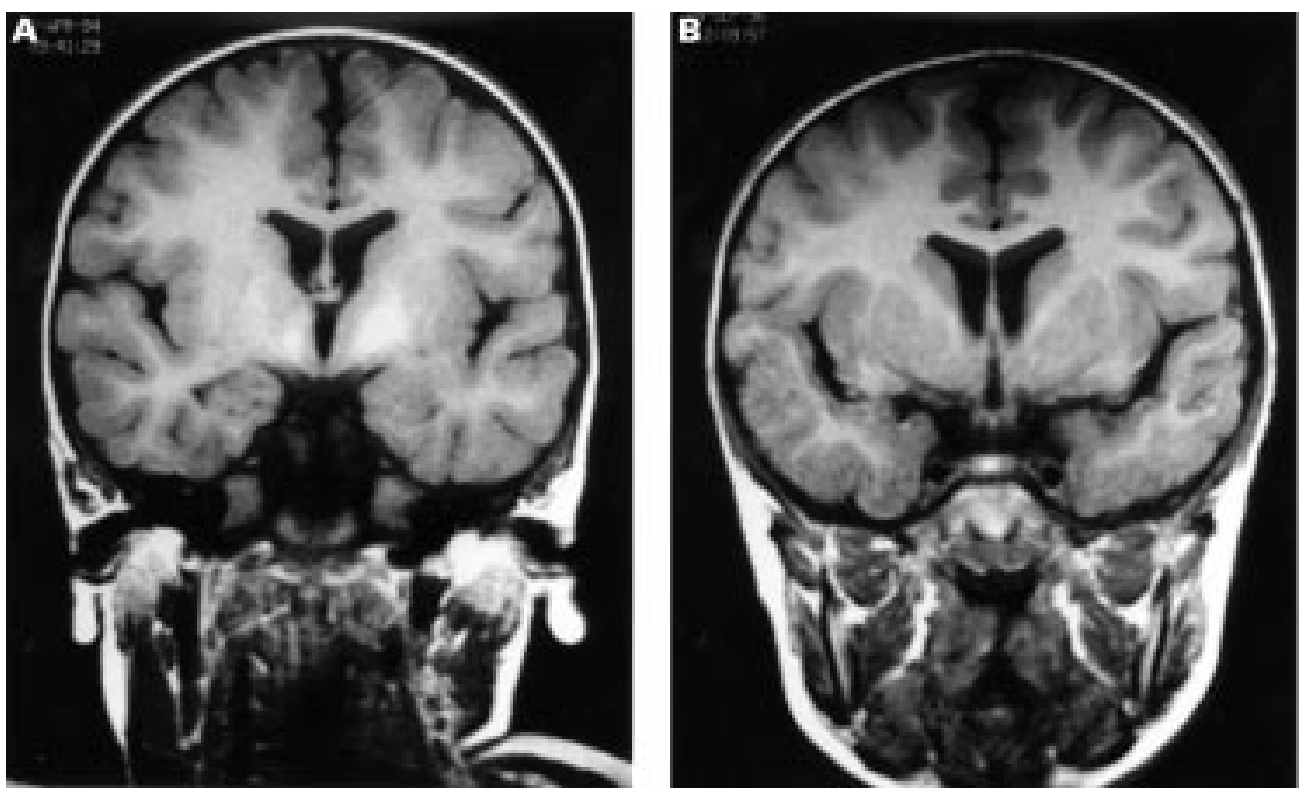

Figure 1 Coronal T1 weighted MRIs in case 1. (A) Abnormal high signal in globus pallidus. (B) Regression of abnormal signal after reduced manganese supplementation.

istration, the child was evaluated regularly. $\mathrm{He}$ had periodic clinical, neurological, and developmental assessment as well as measurement of whole blood manganese concentrations, liver function, and trace elements. A cranial MRI was repeated each year with parameters identical to those used previously. To compare the serial MRIs objectively the percentage of contrast was used as an intensity index as previously described. ${ }^{7}$ The signal intensity value of cortex (C) was subtracted from the mean signal intensity value of globus pallidus (GB) and divided by $\mathrm{C}$ : percentage of contrast $=(\mathrm{GB}-\mathrm{C}) / \mathrm{C} \times 100$.

During three years of follow up neurological and developmental examinations remained normal. The oral feeds, parenteral nutrition requirements, main findings of the laboratory investigation, as well as the MRI measurements are shown in table 1 . There were no significant changes in nutritional status or liver function during this time. However whole blood manganese concentrations gradually fell and the MRI index progressively decreased from 0.318 to 0.205 . The MRI changes previously observed in the basal ganglia clearly regressed and the image became indistinguishable from normal (fig 1).

Table 1 Main characteristics and follow up findings in two siblings with enteropathy of unknown cause

\begin{tabular}{llllll}
\hline & $\begin{array}{l}\text { Parenteral } \\
\text { nutrition } \\
\text { (kcal/kg/day) }\end{array}$ & $\begin{array}{l}\text { Enteral nutrition } \\
\text { (kcal/kg/day) }\end{array}$ & $\begin{array}{l}\text { AST } \\
\text { (U/l) }\end{array}$ & $\begin{array}{l}\text { Blood manganese } \\
\text { (nmol/l) }\end{array}$ & MRI index \\
\hline Case 1 & & & & & \\
1994 & 55 & 50 & 26 & 323 & 0.318 \\
1995 & 48 & 30 & 31 & 373 & 0.320 \\
1996 & 44 & 50 & 28 & 200 & 0.201 \\
1997 & 49 & 45 & 37 & 226 & 0.205 \\
Case 2 & & & & & \\
1994 & 66 & 10 & 31 & 516 & 0.153 \\
1995 & 60 & 15 & 28 & 135 & 0.134 \\
1996 & 57 & 20 & 21 & 209 & \\
1997 & 64 & 20 & & &
\end{tabular}

AST, aspartate aminotransferase; NA, not available.
Case 2

The sibling of case 1 was admitted at the age of 1 month with the same condition as her brother. Inpatient management consisted of total parenteral nutrition while enteral feeding was instituted gradually. At 2 years of age (23 months on parenteral nutrition) she was also found to have raised whole blood manganese at $516 \mathrm{nmol} / \mathrm{l}$. Liver function tests and serum iron, ferritin, copper, zinc, and selenium were all within normal limits. Similarly, brain MRI scan showed symmetrical high signal changes in the basal ganglia on T1 weighted sequences. Like her brother, she was neurologically and developmentally normal. As a consequence of these findings, the manganese in the parenteral nutrition was also changed. She was regularly monitored in the same fashion as her brother and the main findings are shown in table 1.

\section{Discussion}

The recognition that manganese is an essential trace element required for mucopolysaccharide production and the formation of normal bone, cartilage, and connective tissue, led to its incorporation into parenteral solutions, although the exact daily requirements were not well understood. ${ }^{8}$ Normally, an efficient regulatory mechanism prevents excessive absorption of ingested manganese from the gut as less than $3 \%$ is absorbed and only $1 \%$ is retained after normal biliary excretion. ${ }^{4}$ Conversely, in patients on parenteral nutrition this normal homeostatic control mechanism is bypassed allowing for excessive absorption and tissue deposition of manganese. As a result, hepatic and nervous system diseases have been associated with manganese toxicity in patients on parenteral nutrition. ${ }^{29}$ Severe neurological disorders such as seizures and dystonic movements have been described in patients with extremely high blood manganese concentrations and have had a fatal outcome. ${ }^{12}$ Manganese has 
a special affinity for the nerve tissue of the extrapyramidal system and this can be seen by MRI. The sites of the earliest and most profound manganese deposition are the basal ganglia, especially the globus pallidus, because of the high density of dopamine receptors in these areas. Animal studies have shown that manganese binds to dopaminergic receptors causing auto-oxidation of dopamine and possibly formation of local catecholamines and free radicals. ${ }^{10}$

However, MRI changes are not always associated with neurological manifestations. We report two children on long term parenteral nutrition in whom manganese neurotoxicity was detected with abnormal whole blood manganese concentrations and MRI signal alteration. Despite positive MRI findings and moderately raised whole blood manganese, the children were neurologically normal without any overt developmental disorder. This is probably indicative of subclinical manganese toxicity; presumably in severe toxicity with dystonia there is major disruption of dopamine transmission in the basal ganglia and tissue destruction from the free radical production.

It is unclear from the literature whether manganese can be cleared from brain tissue. Similarly it is not clear whether manganese related disorders are progressive even when exposure has ceased. Manganese has a half life of 10-42 days in blood but this is substantially prolonged if the metal has accumulated in the central nervous system. ${ }^{11}$ Persistent and progressive neurological abnormalities have been described in adults on parenteral nutrition even where exposure to manganese has been stopped. $^{12}$ A complete resolution of neurological symptoms has been reported with reversal of basal ganglia alterations in the MRI after discontinuation of parenteral manganese. ${ }^{59}$ Radiological findings improved after 22 weeks and one year of manganese withdrawal.

Despite the neurotropic nature of manganese and unlike the above mentioned adult experience, the children in our study did not present any neurological signs and developed normally during follow up. Whole blood manganese gradually fell and the abnormal MRI features regressed. The decrease in whole blood manganese appeared to parallel the change in MRI indices during follow up. ${ }^{13}$

In conclusion, our cases show that manganese deposited in the brain may not result in clinically overt neurological and developmental disorders and that this manganese can be slowly cleared. Finally, as manganese deposition can occur asymptomatically and whole blood manganese concentration is a good indicator of manganese exposure and toxicity, children on long term parenteral nutrition should have regular monitoring of manganese concentrations.

We are grateful to Dr Kling Chong for assistance with MRI imaging.

1 Reynolds AP, Kiely E, Meadows N. Manganese in long term paediatric parenteral nutrition. Arch Dis Child paediatric paren $1994 ; 71: 527-8$.

2 Fell JME, Reynolds AP, Meadows N, et al. Manganese toxicity in children receiving long-term parenteral nutrition. Lancet 1996;347:1218-21.

3 Ono J, Harada K, Kodaka R, et al. Manganese deposition in the brain during long-term total parenteral nutrition. $\mathcal{F}$ Parent Ent Nutr 1995;19:310-12.

4 Quaghebeur G, Taylor WJ, Kinsgley DPE, Fell JME, Reynolds AP, Milla PJ. MRI in children receiving total parenteral nutrition. Neuroradiology 1996;38:680-3.

5 Mirowitz SA, Westrich TJ. Basal ganglial signal intensity alterations: reversal after discontinuation of parenteral manganese administration. Radiology 1992;185:535-6.

6 Committee on Clinical Practice Issues of the American Society for Clinical Nutrition. Guidelines for pediatric parenteral nutrition. Am f Clin Nutr 1988;48:1324-42.

parenteral nutrition. Am f Clin Nutr 1988;48:1324-42.
7 Mirowitz SA, Westrich TJ, Hirsch JD. Hyperintense basal Mirowitz SA, Westrich TJ, Hirsch JD. Hyperintense basal
ganglia on T1-weighted MR images in patients receiving parenteral nutrition. Radiology 1991;181:117-20.

8 Dahlstrom KA, Ament ME, Medhin MG, Meurling S. Serum trace elements in children receiving long-term parenteral nutrition. $\mathcal{F}$ Pediatr 1986;109:625-30.

9 Ejima A, Imamura T, Nakamura S, et al. Manganese intoxication during total parenteral nutrition [letter]. Lancet 1992;339:426.

10 Mergler D. Manganese: the controversial metal. At what levels can deleterious effects occur? Can $\mathcal{F}$ Neurol Sci 1996; 23:93-4.

11 Krieger D, Krieger S, Jansen $\mathrm{O}$, et al. Manganese and chronic hepatic encephalopathy. Lancet 1995;346:270-4.

12 Alves G, Thiebot J, Tracqui A, et al. Neurologic disorders due to brain manganese deposition in a jaundiced patient receiving long-term parenteral nutrition. F Parent Ent Nutr receiving long

13 Houser RA, Zesiewicz T, Martinez C, Rosemurgy A, Olanow C. Blood manganese correlates with brain magnetic resonance imaging changes in patients with liver disease. Can f Neurol Sci 1996;23:95-8. 